\title{
Establishment of Paediatric Airway Surgery Services in Tribhuvan University Teaching Hospital: A Leap in Otorhinolaryngology in Nepal
}

\author{
Rajendra Guragain $^{1} \cdot$ Bigyan Raj Gyawali $^{1}\left(\mathbb{D} \cdot\right.$ Heempali Dutta $^{1} \cdot$ Yogesh Neupane $^{1}$
}

Received: 22 January 2019/Accepted: 11 July 2019/Published online: 25 July 2019

(C) Association of Otolaryngologists of India 2019

\begin{abstract}
Paediatric airway surgery is an emerging superspeciality for a developing country like Nepal. Thriving in a resource limited environment of the country, Tribhuvan University Teaching Hospital is setting its early steps in this subspecialty of paediatric otorhinolaryngology. With generous support of the experts from USA and a team of very dedicated surgeons in the department, future of paediatric airway surgery in Nepal seems to be promising. Here, we discuss in brief about the establishment of paediatric airway surgery in Tribhuvan University Teaching Hospital, the tertiary referral center of Nepal with short discussion on the cases operated so far and their outcomes.
\end{abstract}

Keywords Paediatric · Airway surgery ·

Tribhuvan university $\cdot$ Nepal

\section{History}

Paediatric airway surgery started more than 100 years ago. Von Schroetter, an Austrian laryngologist, attempted the first documented case of laryngeal dilation using tin bolts

Bigyan Raj Gyawali

bigyan.gyawali@gmail.com

Rajendra Guragain

professorrajendraguragain@gmail.com

Heempali Dutta

heempalidutta@hotmail.com

Yogesh Neupane

yogeshneupane@gmail.com

1 Department of ENT-HNS, Institute of Medicine, Kathmandu, Nepal and hard India rubber in the 1870s [1]. Surgeons such as O'Dwyer and Lefferts, soon followed his lead and reported success with dilation and intubation [2].

Along with introduction of paediatric endotracheal intubation, the incidence of subglottic stenosis increased in children in the 1960s [3]. The incidence peaked in the late 1970s and early 1980s [4-6] and this was the period when various open surgical procedures came forth. Ruggi, an Italian surgeon, performed an open surgical procedure aimed at correcting laryngeal stenosis [7].

Nepal, in its budding phase of paediatric airway surgery and critical care medicine, faces lot of challenges at current period. Institute of medicine, Tribhuvan Universirty Teaching Hospital, is the leading institution among the tertiary centers in the country. Department of Otorhinolaryngology and Head and neck surgery of IOM has been the pioneer in establishing the paediatric airway surgery in Nepal. Though procedures such as cold steel excision of respiratory papillomatosis and rigid bronchoscopy were being done since earlier days of establishment of the department, history of paediatric airway surgery at our centre dates back to May 2014. A paediatric airway workshop course was organized for the first time under the initiative of Prof. Rajendra Guragain, the then Head of Department and Unit Chief of Paediatric Otorhinolaryngology, with Dr. Ajoy Mathew Varghese, a renowned airway surgeon from Vellore, India being invited as an external faculty. Dissection in the cadaver of procedures like tracheostomy, closure of tracheostomy, anterior and posterior cricoid split with rib graft, laryngofissure and partial cricotracheal resection and anastomosis were demonstrated. Surgeries like microlaryngoscopic release of anterior glottic web, removal of laryngeal papillomatosis by microdebrider and cricofissure with costal cartilage graft were performed. It was the period when the first 
milestone was set. Following this initiation, further paediatric airway workshops were organized in 2016/17. This time Prof. Lary Hoover, Dr. Joseph Edmonds and Prof. Dinesh Chettri with his team of Dr. Karthik Balakrishnan and Dr. Douglas Ryan Sidell from United States of America took keen interest for further development of the services by providing their expertise and helping out with surgical equipments. Various aspects of paediatric airway surgery were explored such as endoscopic evaluation, endoscopic balloon dilatation, cricoid split with graft including different approaches of anesthesia. These activities provided opportunity for training the junior faculties in paediatric airway surgery. It was the time when $\mathrm{CO} 2$ LASER came into operation for the first time in Nepal, being used for excision of subglottic stenosis and arytenoidectomy.

\section{Cases}

Here we present a case series of airway problems operated so far at our center.

\section{Case 1}

A 8 months old male BS was presented by his parents with a history of noisy breathing associated with indrawing of chest and cyanosis, which was increased on crying and agitation. Flexible nasopharyngolaryngoscopy (NPL) revealed flabby epiglottis and arytenoids and emergency tracheostomy was done. He underwent supraglottoplasty 4 months later. Endoscopic evaluation following surgery showed minimal redundant mucosa over bilateral aryepiglottic folds with normal glottis and subglottis and minimal suprastomal granulation. Three months following supraglottoplasty child was re-operated for excision of suprastomal granulation. Following uneventful corking of tracheostomy overnight, child was decannulated the following day. After observation for couple of days, child was discharged from hospital.

\section{Case 2}

$\mathrm{NH}$, a 9 months old female who fell from 10 feet height following which she sustained brain injury (left frontalparietal subdural hematoma with right cerebral infraction). During the treatment course, she was intubated in PICU for 24 days. Repeated failures of extubation ended her up with tracheostomy. Tracheoscopic evaluation was done on a later date, which showed grade IV stenosis of subglottis and trachea being normal. She underwent balloon dialation with application of Mitomycin $\mathrm{C}$ for 4 consecutive sessions 3 weeks apart over a duration of 3 months. Due to inadequate widening of stenotic segment (grade III) despite repeated dialation, an attempt of an open procedure was decided once the child was 18 months. Dialation of the stenosis with anterior and posterior cricoid graft was done with placement of stent. Stent was removed after 2 weeks followed by successful decannulation of the child.

\section{Case 3}

LS, a 7 years old male was presented with 3 days history of shortness of breath and snoring during sleep. Flexible nasopharygolarygoscopic evaluation was done which showed bilateral abductor palsy. He underwent emergency tracheostomy on the same day and was then discharged on a nonmetallic tracheostomy tube. Rigid endoscopy was done 6 months later which showed partially abducted vocal cords with adequate glottic chink. The child was planned for decannulation, however 2 weeks following decannulation he was again presented by his parents with persistent stridor during sleep. Left LASER arytenoidectomy was done following tracheostomy. Child was re-evaluated after 6 weeks and decannulation was attempted, but child developed dyspnea and didn't tolerate corking. Sleep endoscopy was performed which showed laryngomalacia causing the stridor during sleep with adequate glottic chink. LASER supraglottoplasty was performed. Child was then re-evaluated after 3 weeks when the corking was performed followed by successful decannulation.

\section{Case 4}

SK, a 6 years female developed progressive shortness of breath following prolonged intubation for Guillain Barre syndrome and was diagnosed as a case of subglottic stenosis (grade III) at our center. She was tracheostomized immediately to secure airway. LASER excision of stenosis with balloon dialation with application of Mitomycin C was done. She was evaluated 3 months later endoscopically. Stenosis improved to grade I and thus was decannulated after uneventful corking overnight.

\section{Case 5}

$\mathrm{SB}$, a 8 years old male and a known case of recurrent respiratory papillomatosis, was operated 7 times at our center. He had complaints of persisternt hoarseness and dyspnea on exertion. Anterior glottic web was recognized on NPL and cold steel excision of the web was done with application of Mitomycin C. The patient later was lost to follow up. 


\section{Case 6}

$\mathrm{SN}$, a 3 years old male child was brought by his parents with a history of accidental ingestion of LED battery. Rigid oesophagoscopy and foreign body removal was done within $7 \mathrm{~h}$ of ingestion. Foreign body was present at cricopharyngeal junction. After 10 days of hospital stay, patient developed noisy breathing. NPL evaluation showed bilateral vocal cord paralysis and emergency tracheostomy was done. Endoscopic evaluation was done 8 months later but bilateral abductor palsy was still present. Small diverticulum was seen in right postcricoid region however, insufflation test showed no tracheoesophageal fistula. He was re-evaluated 3 months later and this time vocal cords were partially mobile with adequate glottic chink. Decannulation was attempted, but the child didn't tolerate the procedure and a re-tracheostomy had to be done. LASER right cordotomy with arytenoidectomy with suture lateralization of right cord was done with suprastomal suspension of malatic segment of anterior tracheal wall. Following the procedure child was successfully decannulated after 3 months. However, the child has developed tracheocutaneous fistula and is planned for the closure.

\section{Case 7}

AS, a 15 years old male, being operated 27 times for juvenile recurrent respiratory papillomatosis, presented with persistent hoarseness and progressive dyspnea. Anterior glottic web was seen on NPL evaluation. He underwent $\mathrm{CO} 2$ LASER excision of web with application with Mitomycin C. However, he was lost to follow up.

\section{Case 8}

RS, a 4 years old male, presented with an insidious onset progressive noisy breathing. He was diagnosed as a case of bilateral abductor palsy following NPL. Tracheostomy was done and the child was discharged on a metallic tracheostomy tube. Endoscopic evaluation done 18 months later revealed persistent bilateral abductor palsy. Posterior cricoid graftof rib cartilage was done and the child was discharged on metallic tracheostomy tube after few days of observation. 2 months later he was re-evaluated. The glottic chinck was adequate and graft was in situ. He tolerated the corking overnight and was decannulated the other day. On follow up the child doesn't have any complaints and has a good voice.

\section{Case 9}

EG, a 18 months male was brought by his parents with a history of progressive hoarseness and dyspnea for
3 months. NPL evaluation revealed multiple papillomatous growth arising from left false vocal cords, true vocal cords and anterior commissure. Removal of papillomatous growth was done using microdebrider. The child was discharged the other day and followed up after 3 months. He had a good voice with no papillomatous growth on endoscopic evaluation. The child is now under regular follow up.

\section{Case 10}

EG, a 4 years old male child, developed change in voice and noisy breathing at the age of 9 months after being treated in ICU for anemia with protein energy malnutrition with acute laryngitis however, he had no history of intubation during ICU stay. He was diagnosed of having anterior glottic web on endoscopic evaluation and underwent elective tracheostomy at 14 months of age. Cold steel excision of the web with balloon dilatation was done after $2^{1 / 2}$ years. He was then evaluated after 6 months and decannulation was done. Child, however had a supraglottic voice and was planned to manage conservatively.

\section{Case 11}

BKC, a 13 months old female child, was consulted by the paediatric team at our center for progressive respiratory distress. She was a diagnosed case of interstitial lung disease. She had a history of being intubated in paediatric ICU for 2 weeks. Endoscopic evaluation revealed circumferential glottic web with a very narrow glottic chink. Despite balloon dialation for 3 times consecutively, web persisted. She has been planned for posterior cricoid split with graft.

\section{Case 12}

JS, a 12 years old male child, was brought by his parents with the complaints of change in voice for past 8 months. He had mild dyspnea on exertion but no ovious respiratory difficulty, stridor or cyanosis. NPL evaluation showed multiple papillomatous growth involving left TVC and anterior commissure. Excision of growth via microdebrider was done. The child is under regular follow up with no major complaints so far.

\section{Discussion}

The emergence of the superspeciality of paediatric otolaryngology and airway surgery is not very old. Even in the developed countries like USA and UK the services are provided only by few centers. Operating in children is a very sensitive issue and managing them well is very 
challenging. A good paediatric airway surgery center must have a dedicated team of paediatric otolaryngologists, paediatric anaesthesiologists, well trained nursing staffs and well equipped intensive care with dedicated staffs.

Paediatric airway surgery is an emerging superspeciality for a country like Nepal. Being a developing country it has yet not progressed in the field of education and health services. In the remote areas of country where people still struggle the absence of proper primary health services along with maternal and child health care, problems such as premature delivery, low birth weight, malnutrition etc. are still prevalent. Cases of child sustaining injury to the airway are increasing. On the other hand there are poor intensive care facilities with lack of standardized protocols. All these factors have led to emergence of airway problems such as laryngomalacia, respiratory papillomatosis, airway stenosis etc. In this scenario, paediatric airway surgery specialization has a lot to offer.

Department of ENT-Head \& Neck surgery of Institute of Medicine, Tribhuvan University Teaching Hospital has been the only place where paediatric airway surgery is being practiced at the current period in Nepal. With the generous support of the experts from outside the country, both on logistics and technical grounds, this department has progressed drastically within a period of few years in this field. Though the open surgical procedures are still challenging, endoscopic procedures like web excision using LASER or cold steel, balloon dilatation of laryngeal stenosis, microdebridement of papillomatosis are being done on a weekly basis. With ongoing paediatric airway workshops being held 2-3 times a year, various aspects of paediatric airway surgery are yet to be explored and dynamicity of surgical expertise seems to be widening.

Despite being a tertiary center, several challenges are, however, still present. Though various logistic supports have been offered from within and outside the institution, there is still lack of proper instruments and a suitable operation theatre set up. Intensive care facility is another biggest problem we are facing currently. Lack of adequate intensive care unit beds has been restraining the number of operations to reach optimally and on the other side, poor post operative care such as tracheostomy tube care have been resulting in unanticipated morbidities and mortalities.
With the existence of Paediatric Otolaryngology unit in the Tribhuvan University Teaching Hospital and the keen interest of hard working dedicated team in the unit, the future of paediatric airway surgery services seems to be bright.

\section{Conclusion}

Paediatric airway surgery is an emerging superspeciality in Nepal. With the support of foreign experts, Department of ENT-Head and Neck Surgery of Institute of Medicine, Tribhuvan University Teaching Hospital has thrived in various aspects of paediatric airway surgery. Further exposure in this field and improvement in intensive care facilities will hopefully lead this institution to one of the best referral centers for paediatric airway surgery.

\section{References}

1. LeJeune F, Owens N (1935) Chronic laryngeal stenosis. Ann Otol Rhinol Laryngol 44:354-363

2. O'Dwyer J (1897) Congenital stenosis of the larynx. Trans Am Pediat Soc 9:180

3. McDonald IH, Stocks JG (1965) Prolonged nasotracheal intubation. A review of its development in a pediatric hospital. $\mathrm{Br} \mathrm{J}$ Anaesth 37:161-173

4. Cotton RT (1996) Management and prevention of subglottic stenosis in infants and children. In: Bluestone CD, Stool SE, Kenna MA (eds) Pediatric otolaryngology. WB Saunders, Philadelphia, pp 1373-1389

5. Zalzal G, Cotton RT (1998) Glottic and subglottic stenosis. In: Cummings CW, Frederickson JM, Harker LA, Krause CJ, Schuller DE, Richardson MA (eds) Otolaryngology head and neck surgery, vol 5, 3rd edn. Mosby, St. Louis, pp 303-324

6. Lusk RP, Woolley AL, Holinger LD (1997) Laryngotracheal stenosis. In: Holinger LD, Lusk RP, Green CG (eds) Pediatric laryngology and bronchoesophagology. Lippincott-Raven, Philadelphia, pp 165-186

7. Jackson C (1904) Laryngostomy. Ann Otol Rhinol Laryngol 13:690-702

Publisher's Note Springer Nature remains neutral with regard to jurisdictional claims in published maps and institutional affiliations. 